Giuseppe Milan*

ORCID: 0000-0002-1081-1750

Padova, Italia

\title{
Costruire l'intercultura in tempi difficili. La proposta pensiero-vita di Chiara Lubich
}

\section{Building Interculturality in Difficult Times. Chiara Lubich's "Thought-Life" Proposal}

Summary: The thought and action of Chiara Lubich are very important for intercultural education. She has given us many theoretical and practical tools - reinforced by the testimony lived with the greatest coherence - to learn to be intercultural as people, but also as groups, as specific collective and cultural identities. Her experience can be told as an experience of education in dialogue, a micro-macro dialogue that unfolds from the immediate challenge of interpersonal relationships to widen to the areas of political, intercultural, interreligious relations. This article analyses some aspects of today's multicultural reality, which challenges the world of education, and - assuming the demanding perspective of interculturality - examines some of Chiara's proposals, which are based on the idea of a person-dialogue, person-relationship and intercultural person. These proposals, methodologically essential in the transition from reality-challenge to the goals indicated by interculturality, are effectively communicated by Lubich in his fundamental book "The Art of Loving", in which she presents the secrets for interpersonal, social, intercultural relations, the

* Giuseppe Milan, Professore ordinario di Pedagogia interculturale. Docente all'Università degli Studi di Padova e all'Istituto Universitario Sophia a Loppiano (FI). Indirizzo: FISPPA - Via Beato Pellegrino 28, Padova; mail: giuseppe.milan@unipd.it. 
inescapable attitudes that are configured as "concrete love". Some passages are here quoted that present the pedagogical principles of reference according to Chiara $\mathrm{Lu}$ bich, taken from the lecture entitled "Uomo-Mondo" (1972), from the lecture held in Paris on December 17, 1996, when she received the UNESCO prize for "Peace Education", from the lectio magistralis held in Washington on $10^{\text {th }}$ November 2000 (Honorary doctoral Degree in Education from The Catholic University of America).

Keywords: intercultural education; dialogue; multiculturality; community; pedagogy of unity.

Sommario: Il pensiero e l'opera di Chiara Lubich sono molto importanti per l'educazione interculturale: lei ci offre molti contributi teorici e pratici - rinforzati dalla testimonianza vissuta con massima coerenza - per imparare ad essere interculturali come persone, ma anche come gruppi, come specifiche identità collettive e culturali. Tutta la sua esperienza può essere raccontata come esperienza di educazione al dialogo, un dialogo micro-macro che si esplica fin dalla immediata sfida della relazione interpersonale per allargarsi agli ambiti sempre più vasti delle relazioni politiche, interculturali, interreligiose. Questo articolo analizza alcuni aspetti della odierna realtà multiculturale, che sfida il mondo dell'educazione, e - assumendo l'impegnativa prospettiva dell'interculturalità - esamina alcune proposte di Chiara: sono proposte che si fondano sull'idea di persona-dialogo, persona-relazione, persona interculturale. Queste indicazioni di percorso, metodologicamente essenziali nel passaggio dalla realtà-sfida alle finalità indicate dall'interculturalità, sono efficacemente indicate dalla Lubich nel suo fondamentale scritto L'arte di amare, nel quale presenta i segreti per la relazione interpersonale, sociale, interculturale, gli atteggiamenti imprescindibili che si configurano come "amore concreto". Vengono qui riportati alcuni brani che presentano i fondamentali principi pedagogici di riferimento per Chiara Lubich, tratti dal testo di una relazione intitolata "Uomo-Mondo"(1972), dal discorso a Parigi il 17 dicembre 1996, in occasione del conferimento del Premio UNESCO per l'educazione alla pace, dalla lectio magistralis tenuta a Washington il 10 novembre 2000 (Laurea honoris causa in Pedagogia).

Parole chiave: educazione interculturale; dialogo; multiculturalità; comunità; pedagogia dell'unità.

Nessuno mette in dubbio che le nostre società siano oggi multiculturali, che vedano cioè - anche per effetto delle migrazioni - la compresenza di 
persone, gruppi, comunità di diverse origini culturali: questa multiculturalità è un dato strutturale, non è tanto un'emergenza (come spesso si dice) ma è la realtà oggettiva, indiscutibile, che caratterizza la convivenza degli esseri umani, ormai a tutte le latitudini. Certamente è "realtà-problema", che sfida in particolare il mondo dell'educazione. Ritengo infatti che proprio l'educazione abbia il compito essenziale di indirizzare la realtà odierna verso l'orizzonte dell'intercultura, rendendo l'intercultura stessa - cioè il dialogo concreto, creativo, tra quelle che oggi sono "isole" separate dell' "arcipelago multiculturale" - non soltanto una visione ideale ma una realtà già presente in mezzo a noi. L'intercultura, in questa prospettiva, si pone sul piano dell " "utopia", intesa non come un'illusione ma come l'isola che ancora non c'è ma che potrebbe e dovrebbe esserci, alla quale possiamo e dobbiamo avvicinarci: è sempre "incompiuta", un orizzonte cui tendere, un "cantiere sempre aperto" che comunque costruisce qualcosa di nuovo con i piccoli umili passi quotidiani, i passi dell'educazione.

A tal fine un ruolo insostituibile è quello dei grandi "testimoni", cioè di quelle personalità capaci di indicarci e di avvicinarci alla meta, di darci una bussola utile per farci camminare, portando un originale e creativo contributo di pensiero e di azione.

Una figura di grande importanza per l'intercultura è certamente Chiara Lubich (1920-2008), una vera Maestra in questa prospettiva: ci ha dato molti strumenti teorici e pratici - rinforzati dalla testimonianza vissuta con massima coerenza - per imparare ad essere interculturali come persone, ma anche come gruppi, come specifiche identità collettive e culturali. Tutta la sua esperienza può essere raccontata come esperienza di educazione al dialogo, un dialogo micro-macro che si esplica fin dalla immediata sfida della relazione interpersonale per allargarsi agli ambiti sempre più vasti delle relazioni politiche, interculturali, interreligiose.

Ricordo che a Washington, il 10 novembre 2000, in occasione del conferimento della laurea honoris causa in Pedagogia, Lubich ha iniziato la sua lezione affermando che "Il nostro Movimento e la nostra storia possono essere visti come un grande, straordinario evento educativo" potremmo dire che "il nostro Movimento e la nostra storia - proprio grazie al carisma di Chiara Lubich - sono un grande, straordinario evento interculturale". Molte sono le esperienze che, su iniziativa del Movimento dei Fo-

${ }^{1}$ Chiara Lubich, "Lezione per la laurea honoris causa in pedagogia, Washington 10 novembre 2000”, Nuova Umanità 135/136 (2000): 341-352. 
colari, sono veri e propri "luoghi interculturali". Basti pensare, solo, per fare alcuni esempi, alle molte scuole che a livello-mondo si ispirano all'ideale di Chiara Lubich $^{2}$. Basti pensare, ancora, alle innumerevoli esperienze di dialogo interreligioso-interculturale attuate frequentemente nei contesti più vari ${ }^{3}$. E d'altronde evidente che queste proposte ed esperienze non sono riservate ai membri del Movimento: esse si rivolgono a ogni essere umano, a ogni realtà culturale, e lo dimostra la loro diffusione a livello mondo.

Fatta questa premessa, mi propongo di esaminare più in dettaglio questo tema, tentando anche di mettere in luce i fondamenti e le implicazioni esistenziali-esperienziali che derivano dalla visione ideale e dall'impostazione pedagogica di Chiara Lubich.

\section{Un primo livello di analisi. Aspetti-sfida della realtà e proposte di Chiara Lubich}

Possiamo innanzi tutto chiederci: in che senso stiamo parlando della multicultura come di una "Realtà-sfida"? Quali sono alcuni elementi di questa Realtà e quali risposte possiamo trovare nel pensiero e nell'opera di Lubich? In che senso queste risposte aprono alla prospettiva dell'intercultura?

Ecco in sintesi alcuni punti-realtà da considerare, rispetto ai quali accostiamo, seppure in sintesi, alcune linee di pensiero-azione indicate da Lubich:

1) Realtà: la multicultura si manifesta spesso in contesti chiaramente anche se non esclusivamente - contrassegnati da individualismo, egocentrismo, sgretolamento sociale, sospetto-paura nei riguardi dell'“altro-straniero", visto spesso come nemico. Sono tendenze culturali certamente presenti nel nostro mondo occidentale, come denunciano molte ricerche, ma che sembrano ormai diffondersi a più ampio raggio, facilitate anche dalla globalizzazione economica e dalle sue conseguenze di tipo culturale. Similmente, a livello teorico, il multiculturalismo-comunitarismo accentua la difesa delle diversità, fino a ritenere negativo o del tutto ingiustificato il "meticciamento culturale", assolutizzando perciò il relativismo culturale e, con questo, il re-

2 Tra le molte scuole che si ispirano alla "pedagogia di comunione" cito le seguenti: Scuola Fantasy (Serbia), Zraka Sunca (Croazia), Biseri (Macedonia), Diamante e Santa Maria (Messico), Aurora e Santa Maria (Brasile), Aurora de un mundo nuevo (Argentina), Café con leche (Rep. Dominicana), Sol Naciente (Colombia).

${ }^{3}$ Cf. Mariola Kozubek, "Dialog międzyreligijny w życiu i działalności Ruchu Focolari”, Paedagogia Christiana 1/27 (2011): 99-117, doi: http://dx.doi.org/10.12775/PCh.2011.020 
lativismo etico (negando perciò la possibilità di un comune riconoscimento di valori e di diritti-doveri trasversali e universali).

Lubich: nessuno mi è straniero, ogni essere umano è l'altro-fratello degno di essere amato concretamente, perché è mio simile, anzi, perché vedo in lui Cristo. L'amore concreto, che si fa dialogo e relazione creativa, vede in ciascuno un fratello. In particolare, il comando evangelico di "amare il nemico" è parte essenziale del pensiero di Lubich sulla relazione umana e sulla realtà della spiritualità collettiva, che si costruisce attraverso quella pedagogia di comunione che implica altruismo, attenzione all'impegno sociale, costruzione attiva del tessuto comunitario, della città nuova e, da qui, di quell'umanità nuova che implica superamento delle frontiere e consapevolezza di appartenere al mondo e di dover essere "uomo-mondo", persone planetarie $e^{4}$.

2) Realtà: si manifestano varie tipologie di risposta alla realtà multiculturale, tra le quali l'assimilazione, cioè il conformarsi rispetto al modello prevalente, e l'apartheid culturale, che genera frammentazione, ghettizzazione, resistenza culturale. Vengono costituite "comunità a parte".

Lubich: ogni persona umana è unica, portatrice di una propria vocazione nel mondo, come pure unica è ogni realtà culturale, unico è ogni popolo, perfino ogni continente ha un colore identitario e culturale specifico e prevalente, che va amato. In questa prospettiva la proposta di Lubich è senza dubbio contestativa nei riguardi del conformismo culturale, del pensiero unico: la comunità è gioco dinamico delle differenze, è molteplicità che trova comunque nel mondo dei valori il segreto dell'unità, è "comunità delle comunità "': nessuna comunità è "a parte", ogni identità culturale apre i propri confini ed è parte della più ampia comunità inclusiva.

${ }^{4}$ Si ricordano al riguardo i seguenti testi che, seppure in anni successivi rispetto al profetico discorso di Lubich, hanno indicato simili finalità pedagogiche: Ernesto Balducci, L'uomo planetario (Milano: Camunia, 1985). Edgar Morin dice che è necessario "perseguire l'ominizzazione nell'umanizzazione in virtù dell'accesso alla cittadinanza terrestre in una comunità planetaria" [Edgar Morin, I sette saperi necessari all'educazione del futuro (Milano: Raffaello Cortina, 2001), 122].

${ }^{5}$ L'istanza "comunità delle comunità" è indicata (con la dizione latina "communitas communitatum") da Martin Buber, fin dal 1950, nel suo Sentieri in Utopia [Martin Buber, Sentieri in Utopia (Milano: Ed. di Comunità, 1967); cf. Giuseppe Milan, Educare all 'incontro. La Pedagogia di Martin Buber (Roma: Città Nuova, 1994), 134-138]. Il medesimo concetto è presente, in rapporto alla situazione geopolitica attuale [Pasquale Ferrara, La politica inframondiale (Roma: Città Nuova, 2014)]. 
3) Realtà: gli stranieri sono spesso visti, con lenti universalizzanti, come un'entità generica; tra entità culturali diverse si costituiscono facilmente rapporti di potere a-simmetrici ("cultura egemonica" - "cultura subalterna"), up-down, facilitati dai "miti della propria perfezione-superiorità e dell'altrui imperfezione-inferiorità" $\mathrm{o}$, al contrario, da quelli della "propria inferiorità e della superiorità altrui".

Lubich: la reciprocità, fondata sull'amore concreto, implica rispetto dell'altro, superamento della tentazione di superiorità, umile disponibilità all'ospitalità che naturalmente contrasta qualsiasi presunzione per costruire a tutti i livelli relazioni capaci di "farsi uno", di empatizzare, vedendo i rapporti tra culture diverse non nel senso della colonizzazione - come spesso avviene - ma nella prospettiva di una reciproca inculturazione che arricchisce le identità, anziché impoverirle o annientarle, e proprio nella simmetria di potere trova la base per la costruzione del mondo comune.

4) Realtà: l'esperienza della migrazione è spesso frutto di una progettualità, di una decisione che orienta attivamente ad affrontare un futuro da costruire; va tuttavia riscontrato che un diffuso abuso di assistenzialismo (soprattutto da parte delle istituzioni dei paesi ospitanti) contrasta spesso tale progettualità, inibendo il protagonismo autentico di chi viaggia-emigra-abita: l'assistenzialismo (non accompagnato da un'effettiva intelligenza educativa) facilita frequentemente un passivo accomodamento in posizioni acquisite, del tutto prive di intraprendenza, impedendo alle persone di essere/diventare attori sociali-culturali-politici; l'assimilazionismo, a partire da una concezione di carattere universalistico, assolutizza invece le somiglianze e perciò il conformismo, l'omologazione culturale, col rischio di indurre al "pensiero unico", alla monotonia culturale, con una specie di improbabile ritorno alla "monocultura".

Lubich: ogni persona umana è chiamata ad essere soggetto, per questo va promossa la responsabilità personale e comunitaria. Le cosiddette "relazioni di aiuto" non devono inibire il protagonismo altrui, anzi il loro compito è quello di dare forza (empowerment) alla singolare progettualità dei singoli e dei gruppi. L'esperienza di molti membri del Movimento dei focolari è "esperienza di viaggio", di trasferimento in contesti culturali diversi dal proprio, all'interno dei quali non essere "stranieri passivi" ma "stranieri attivi", capaci di portare miglioramenti e, perciò, di integrarsi creativamente nel nuovo contesto di vita.

5) Realtà: la multiculturalità mette in stretto contatto tradizioni, costumi, abitudini, appartenenze religiose, visioni del mondo, che - costrette ad 
avvicinarsi e a confrontarsi - possono provocare difficoltà e, in taluni casi, forme di ostilità e intolleranza ${ }^{6}$.

Lubich: l'arte del dialogo, fondata sull'amore, comporta effettiva apertura alla dimensione "tra", cioè all'orizzonte dell'intercultura e del dialogo interreligioso: le tradizioni altre, i costumi diversi, le appartenenze religiose differenti, non sono viste come ostacoli, problemi, elementi ostili o che sollecitano ad alzare muri. L'educazione interculturale, come esperienza di autentica reciprocità, agisce anche come prospettiva "per contrasto": capisco meglio me stesso, la mia identità, la mia cultura nella misura in cui ho l'occasione di confrontarmi con l'identità altra e la cultura altra (io mi costruisco attraverso l'altro $)^{7}$.

\section{Possibili strategie per l'educazione interculturale}

L'intenzionalità interculturale si propone un'impresa difficile, l' "unità nella molteplicità", ritenendola possibile, anzi auspicabile nei nostri contesti, a partire dal microcosmo delle nostre relazioni ravvicinate, nei nostri "piccoli mondi" 8 di ogni giorno, fino ad arrivare alla dimensione planetaria.

La proposta di Chiara Lubich vede l'intercultura come progetto-impegno-compito che riguarda evidentemente molti ambiti dell'attività umana, che vanno profondamente rinnovati, tanto che Lubich stessa, nella prospettiva dell'"Umanità nuova", ha promosso linee di ricerca-studio per una "Politica nuova", "Diritto nuovo", "Arte nuova", "Economia nuova", "Medicina nuova" etc.. Potremmo anche accostare all'attributo "nuovo-nuova" quello "di comunione" (es. "Economia di comunione") che, per Chiara, significa anche "interculturale": "Politica interculturale", "Arte interculturale", "Medicina interculturale", etc.

Ed è evidente che assegna un ruolo fondamentale, primario, all'educazione: non si può pensare a costruire la polis interculturale, se non attraverso

${ }^{6}$ Al riguardo, può essere interessante approfondire il concetto di "autoctonìa" [Cf. Mircea Eliade, Miti sogni e misteri (Milano: Rusconi, 1976); Giuseppe Milan, "Dal 'metodo per le vie brevi' al dialogo in profondità e in altezza. Creatività per l'intercultura", Studium Educationis 2 (2015): 115-126; Giuseppe Milan, Margherita Cestaro, We Can Change. Seconde generazioni, Mediazione interculturale, città (Lecce: Pensa MultiMedia, 2016), 18-28].

7 Su questo argomento cfr. Milan, Educare (prima parte).

${ }^{8}$ Cf. Tommaso Sorgi, Costruire il sociale. La persona e i suoi «Piccoli mondi» (Roma: Città Nuova, 1998). 
il rilevante apporto dell' "educazione nuova”, perciò della "pedagogia di comunione", della "pedagogia interculturale".

Possiamo allora chiederci quali siano, nel pensiero e nell'opera di Lubich, alcune specificità rispetto all'impegno pedagogico nella prospettiva dell'intercultura.

Ecco, sempre in sintesi, alcune caratteristiche dell'educazione interculturale:

- Per Lubich l'intercultura è una dimensione propria dell'essere umano visto come dialogo. Perciò, l'educazione interculturale non è tanto una materia tra le altre, una disciplina di settore: l'interculturalità va vista come dimensione trasversale, che riguarda tutto il lavoro educativo. Non si può pensare un'educazione che non sia interculturale (trasversalità dell'intercultura nell'educazione).

- Non è un'attività educativa riservata ad una categoria esclusiva, ad esempio per gli "alunni stranieri a scuola", per le famiglie di origine straniera presenti nel territorio. Essa si riferisce ad un modus vivendi che interessa ogni essere umano, perciò riguarda ognuno e tutti: ogni essere umano ha bisogno di aprirsi all'alterità, all'accoglienza, alla reciprocità, alla solidarietà, alla fraternità (queste sono le "parole-chiave" dell'intercultura).

- Prima di puntare all'intercultura come ad una costruzione "sociale"-"politica"-“culturale"-“organizzativa", la prospettiva pedagogica di Lubich ha come finalità la "persona-dialogo" (teologicamente, potremmo parlare di "persona" che vive "trinitariamente", essendo Cristo, essendo immagine di Dio): il fondamento, il principio-guida pedagogico, è perciò il "soggetto interculturale", capace di dimensione "inter", di porsi come identità dialogica, plurale-singolare, di essere "ospite" nell' andirivieni della reciprocità 9 . Da questa "relazionalità costitutiva" si costruisce perciò l'intercultura, anche a livello "macro", a partire dal "micro", da ciascuno, dal soggetto (questo è lo specifico apporto dell' educazione); si potrebbe affermare che la dimensione antropologica-etica-pedagogica dell'intercultura precede la dimensione culturale-sociale-politica. È veramente di fondamentale importanza, nel pensiero di Lubich, questa determinazione "educativa" che fa immediato e imprescindibile riferi-

${ }^{9}$ Cf. Giuseppe Milan, "Ospitarsi”, in: Intercultura, ed. Anna Granata (Roma: Città Nuova, 2012), 137-150. 
mento alla singolarità della persona, alla responsabilità di ciascuno come base portante della costruzione comunitaria e interculturale.

- Il progetto pedagogico interculturale deve essere "sistemico" e prevedere perciò condivisione-cooperazione-alleanza-rete tra $\mathrm{i}$ fondamentali agenti pedagogici: famiglia, scuola, associazioni, istituzioni, mass-media (sistemicità dell'educazione interculturale) ${ }^{10}$; al riguardo, l'esperienza del Movimento dei Focolari è davvero significativa ed esemplare, in quanto nessuna istituzione, nessuna categoria è esclusa dal compito comune: essere tutti "agenti di sistema", soggetti fondamentali di una progettualità sistemica trasversale, che nel contempo promuove e valorizza i singoli apporti.

- Le culture non sono viste come reificate, monolitiche, ermetiche, impermeabili: similmente alle persone, sono soggette a quei processi di cambiamento che sono provocati specialmente dalle relazioni, dalle costruttive contaminazioni nell'incontro creativo a livello interpersonale, sociale, interculturale; le culture sono perciò porose, sempre in movimento, dinamiche, con i confini permeabili.

- La persona umana, secondo questa prospettiva pedagogica, che è presente in tutta l'opera di Lubich, è vista come l'attore per eccellenza di intercultura: essa è cultura ma non è riducibile deterministicamente ad una cultura, è capace (per la libertà che la contrassegna) di affrancarsi, di distinguersi e di oltrepassare creativamente la sua cultura di appartenenza, rendendola perciò "porosa". Dalla cultura la persona riceve molto, da essa viene condizionata, ma non determinata. Le persone umane, perciò, sono i primi catalizzatori di intercultura: non sono tanto le culture che si incontrano, ma persone di diverse culture che stabiliscono tra loro una comunicazione vitale: è la relazione con l'altro che conta e non la relazione della sua cultura in senso astratto. Questa è l'esperienza che i membri del movimento hanno vissuto, fin dalle origini del movimento stesso, facendo tesoro proprio di questa "plasticità culturale" per "abitare" culture diverse e per arricchirsi esistenziamente di questa componente interculturale.

- L'educazione interculturale evidenzia l'importanza del substrato transculturale: vi è un nucleo di valori comuni condivisibili, che

${ }^{10}$ Una città ben fatta. Il gioco creativo delle differenze, eds. Giuseppe Milan, Emma Gasperi (Lecce: Pensa MultiMedia, 2012). 
Lubich definisce „Semi del Verbo" e che rappresenta la base etica comune a tutti i diversi "mondi". Il lavoro educativo insiste sull'importanza e sulla promozione di questi valori che, insieme alle persone, si pongono come linking agents (agenti di intrecciamento interculturale). Su questo "logos" comune, spesso implicito ma di estrema importanza, si può davvero costruire 1' "Umanità Nuova", che anche da questa radicale appartenenza a valori trasversali - visti non dogmaticamente ma come elementi propulsivi di perenne miglioramento - può e deve trovare "l'unità nella molteplicità".

- L'educazione interculturale promuove il graduale superamento delle distanze interpersonali e culturali, nel passaggio dal livello-base, la tolleranza (che implica la volontà di riconoscere la diversità dell'altro, spesso vissuta come un atteggiamento positivo, ma anche come un vizio), all'accettazione-rispetto della diversità dell'altro, al sostegno reciproco (dove ognuno esprime la stima per l'altro), alla solidarietà-fraternità (attraverso la quale si agisce insieme per affrontare problemi comuni), per arrivare a quella fiducia reciproca che sa anche proporre e accettare la critica reciproca nella realizzazione di una progettualità condivisa.

- Queste indicazioni di percorso, metodologicamente essenziali nel passaggio dalla realtà-sfida alle finalità indicate dall'interculturalità, sono efficacemente comunicate da Lubich nel suo fondamentale scritto L'arte di amare ${ }^{11}$, nel quale presenta i segreti per la relazione interpersonale, sociale, interculturale, gli atteggiamenti imprescindibili che si configurano come "amore concreto".

- In ambito scolastico-didattico la prospettiva interculturale deve prevedere l'interdisciplinarietà (nessuna disciplina è esclusa) e comporta una seria formazione pedagogica per gli educatori, a tutti i livelli.

Le idee-chiave sinteticamente esposte in questo contributo sono presenti e ampiamente argomentate in molti scritti di Lubich, che si pongono come ricchissimi di contributi "interculturali", in dialogo con coerenti impostazioni di altri pensatori. Ricordo, a titolo di esempio, tre brani molto significativi che, in ordine cronologico e a distanza di oltre vent'anni l'uno dall'altro, presentano i principi-guida rappresentativi di questa impostazione culturale-pedagogica:

${ }^{11}$ Chiara Lubich, L'arte di amare (Roma: Città Nuova, 2005). 
1) dal discorso, davvero profetico, "Per un uomo mondo"'l2 tenuto nell'ormai lontano 1972, quando ancora la "sfida dell'intercultura" non si presentava con l'intensità e l'urgenza che avvertiamo oggi:

Nell'epoca della bomba atomica, del frantumarsi di quell'unità che si credeva inscindibile - l'atomo -, il Cielo ci ha fatto scoprire un'atomica divina, una potentissima arma d'amore che, anziché distruggere, anziché radere al suolo l'umanità, la esalti al massimo, dandole la più alta dignità: quella di sentirsi non solo un insieme di popoli giustapposti, che spesso litigano e si minacciano come ragazzoni sgarbati e pericolosi, ma un sol popolo: il popolo di Dio. Un'arma che porti l'unità sulla terra [...]. Voglio parlare della nuova situazione in cui siamo venuti a trovarci dopo l'incontro ormai irreversibile fra i popoli e le civiltà del mondo intero, reso possibile e sollecitato da una vera esplosione dei mezzi di comunicazione sociale e dall'immenso sviluppo tecnologico.

Questo incontro ha portato certamente un gran bene, perché ha fatto circolare notizie, conoscenze, fatti, avvenimenti, tradizioni, mentalità, mettendo tutto in comune, spingendo ogni uomo fuori dalle proprie vedute culturali e nazionali nelle quali era vissuto finora [...]. Ma c'è anche un'altra faccia della medaglia. Non sempre l'uomo di oggi è preparato a questo incontro [...]. Solo adesso cominciamo ad avvicinare quei popoli con il dovuto rispetto, con l'umiltà di chi sa che deve sempre imparare, e per questo ci accorgiamo che c'è tutto un altro modo di vedere le stesse cose, diversissimo dal nostro, di noi occidentali, ma non per questo meno giusto o meno valido [...]. Concezioni, tradizioni, mentalità, nel confronto veloce con altri modi di pensare, altre tradizioni, non resistono. Subentra allora un senso d'incertezza penosa, una sofferenza che l'umanità tutta, più o meno, sente e certamente sentirà allorché i vari punti della terra saranno scossi dall'impatto con altri popoli mai prima conosciuti.

E perché questo? Proprio perché la struttura mentale degli occidentali, il loro modo di pensare, è alle volte così legata con i valori assoluti che quella struttura contiene - come un calice contiene un vino prelibato - che si crede, quando si rompe il calice, che si perda anche il vino; crollando la struttura mentale, si pensa crollino anche i grandi valori umani come l'etica, la morale, l'estetica, la bellezza, la metafisica, la libertà, la giustizia [...]. C'è una risposta pratica, un mezzo sicuro su cui può far calcolo per concorrere con gli altri a generare il mondo che sarà?

C'è in pratica un tipo di uomo-mondo che sente, che ha sentito in sé questo terribile maremoto che minaccia di non salvare nulla di ciò che finora si

${ }^{12}$ Chiara Lubich, Colloqui con i gen, 1970-1974 (Roma: Città Nuova, 1999). 
è creduto intangibile? Che quasi dubita che la stessa verità assoluta lo abbandoni al proprio destino, gettandolo nella più grande confusione? C'è questo uomo-mondo che ha saputo superare tale immane prova, pagando così un mondo nuovo che ha ritrovato in sé e ha generato per gli altri?

Sì, esiste. Ma si intuisce subito che quest'uomo non poteva essere solamente un uomo, ma l'Uomo: è Gesù Abbandonato [...]. A voi conoscerlo profondissimamente, riviverlo...per dare un avvio indispensabile e decisivo alla svolta che l'umanità sta affrontando [...]. A voi accoglierlo nel cuore come la perla più preziosa che oggi vi si possa consegnare, per la vostra anima, per i popoli che qui rappresentate, ma soprattutto per quel mondo nuovo che deve vedere tutti gli uomini uniti. Per quel mondo nuovo che ospiterà non tanti popoli divisi, ma l'unico popolo di Dio ${ }^{13}$.

2) dal discorso tenuto a Parigi il 17 dicembre 1996, in occasione del conferimento a Chiara Lubich del Premio UNESCO per l'educazione alla pace:

Questa spiritualità comunitaria non è legata necessariamente ad una Chiesa: è universale e può dunque essere vissuta da tanti. Per essa, infatti, si sono aperti fecondi dialoghi con tutti gli uomini, con cristiani di molte Chiese, con credenti di diverse Religioni e con persone delle più varie culture, le quali trovano qui sottolineati i valori in cui credono e insieme ci si avvia verso quella pienezza di verità cui tutti tendiamo. Per essa, per questa spiritualità, oggi uomini e donne di quasi tutte le nazioni del mondo, lentamente ma decisamente stanno tentando di essere, almeno là dove si trovano, germi di un popolo nuovo, di un mondo di pace, più solidale soprattutto verso i più piccoli, i più poveri; di un mondo più unito.

\section{3) dalla lectio magistralis tenuta a Washington il 10 novembre 2000:}

La nostra esperienza di vita comunitaria si fonda sull'invito di Gesù: "Amatevi come io vi ho amati... Siate una sola cosa": motivazione questa che è di natura religiosa, ma sono straordinari gli effetti sul piano educativo. La finalità da sempre assegnata all'educazione (formare l'uomo, la sua autonomia) si esplica, quasi paradossalmente, nel formare l'uomo-relazione, che per noi è l'uomo icona della Trinità, capace di autotrascendimento continuo nella realtà di Gesù in mezzo a noi. È attraverso questa prassi spirituale ed educativa dell'amore reciproco, del consumarci in uno - prassi che viene seguita da tutti i membri

\footnotetext{
13 Ibidem, 73-82.
} 
del Movimento, chiamati tutti a vivere l'esperienza comunitaria in piccoli gruppi - che noi operiamo per quella finalità delle finalità, espressa dalla preghiera-testamento di Gesù: "Che tutti siano uno": l'Utopia-Realtà per la quale, come strumenti guidati da Lui, intendiamo spendere la nostra vita [...]. Naturalmente Gesù, che ha saputo compiere questo itinerario pedagogico, questo andirivieni tra l'abbandono e la Trinità, e che, nella sua esperienza terrena, ha vissuto con intensità eccelsa la relazione interpersonale con gli altri, praticando l'empatia, l'accettazione, la speranza, la lotta educativa, la vita di unità col Padre e "con i suoi": Lui è il testimone più autentico e più esigente di cosa significhi essere educatori ${ }^{14}$.

\section{Conclusione}

La prospettiva teorico-pratica che ho presentato in questo scritto richiede senza dubbio un forte impegno trasversale, che chiama in causa sia ciascuno di noi - agenti di trasformazione del nostro mondo - sia le comunità, i gruppi, le diverse identità culturali, i popoli, tutti agenti di relazionalità ad ampio raggio nella prospettiva che vede l'umanità intera come "communitas communitatum". È evidente che siamo tutti implicati da una "vocazione" che viene "dall'alto", come risposta alla chiamata che include l'essere umano nella ineffabile dimensione di una "relazionalità trinitaria", ma che emerge nello stesso tempo "dal basso", come risposta fattiva ad un' umanità che chiede di essere vista come "unità nella molteplicità", come "convivialità delle differenze", perciò come interculturalità.

È una sfida che oggi si pone in termini urgenti e che appare perfino sovversiva - perché destruttura fin dalla radice la consolidata antropologia identitaria individualista e egocentrica e propone il paradigma antropologico identitario dialogico-trinitario-interculturale, che appare davvero innovativo e, perciò, particolarmente difficile da comprendere, da assumere e da vivere.

Credo che proprio l'educazione possa avere oggi, al riguardo, una "funzione risvegliatrice": far prendere atto che c'è il forte rischio, diffuso a livello planetario, di anestetizzazione delle nostre energie spirituali, di sonnambulismo esistenziale, culturale, politico e - in questo contesto alienante - provocare un vero "risveglio" dell'umanità, in ciascuno e fra tutti.

${ }^{14}$ Lubich, "Lezione", 341-352. 
In questa direzione di impegno educativo-culturale-politico c'è un estremo bisogno di testimoni autorevoli e credibili che, con la parola e con la vita, con il pensiero e con l'azione, sappiano aprire strade nuove e, per così dire, fungere da capi-cordata in imprese complesse e difficili. Chiara Lubich ha testimoniato questo e continua a farlo con gli innumerevoli doni di pensiero e di vita che ci ha lasciato. L'intercultura è certamente un progetto incompiuto, che ci provoca e ci sollecita ad avanzare senza sosta in questa prospettiva, anche contro il vento contrario che spesso ostacola il nostro cammino. Abbiamo tuttavia tante risorse ricevute dall'alto, che ci aiutano ad essere, anche come persone interculturali, cercatori di infinito.

\section{Bibliografia}

Balducci, Ernesto. L'uomo planetario. Milano: Camunia, 1985.

Buber, Martin. Sentieri in Utopia. Milano: Edizione di Comunità, 1967.

Eliade, Mircea. Miti sogni e misteri. Milano: Rusconi, 1976.

Ferrara, Pasquale. La politica inframondiale. Roma: Città Nuova, 2014.

Kozubek, Mariola. "Dialog międzyreligijny w życiu i działalności Ruchu Focolari”.

Paedagogia Christiana 1/27 (2011): 99-117. doi: http://dx.doi.org/10.12775/ /PCh.2011.020

Lubich, Chiara. "Lezione per la laurea honoris causa in pedagogia, Washington 10 novembre 2000". Nuova Umanità 135/136 (2000): 341-352.

Lubich, Chiara. Colloqui con i gen, 1970-1974. Roma: Citta Nuova, 1999.

Lubich, Chiara. L'arte di amare. Roma: Città Nuova, 2005.

Milan, Giuseppe, Margherita Cestaro. We Can Change. Seconde generazioni. Mediazione interculturale, città. Lecce Pensa: MultiMedia, 2016.

Milan, Giuseppe. "Dal 'metodo per le vie brevi' al dialogo in profondità e in altezza. Creatività per l'intercultura". Studium Educationis 2 (2015): 115-126.

Milan, Giuseppe. "Ospitarsi”. In: Intercultura, ed. Anna Granata, 137-150. Roma: Città Nuova, 2012.

Milan, Giuseppe. Educare all'incontro. La Pedagogia di Martin Buber. Roma: Città Nuova, 1994.

Morin, Edgar. I sette saperi necessari all'educazione del futuro. Milano: Raffaello Cortina, 2001.

Sorgi, Tommaso. Costruire il sociale. La persona e i suoi «Piccoli mondi». Roma: Città Nuova, 1998.

Una città ben fatta. Il gioco creativo delle differenze, eds. Giuseppe Milan, Emma Gasperi. Lecce: Pensa MultiMedia, 2012. 